\title{
Low HOXC10 expression in liver cancer regulates proliferation via a mechanism involving miR-221 and the MAPK signaling pathway
}

\author{
KEXIN MA ${ }^{1 *}$, CHONGYU ZHAO $^{1 *}, \mathrm{KUN} \mathrm{GUO}^{1}, \mathrm{ZHAOYU} \mathrm{FU}^{1}, \mathrm{CHI} \mathrm{CHE}^{1}, \mathrm{BING} \mathrm{DONG}^{1}, \mathrm{CHONG} \mathrm{PANG}^{1}$, \\ SHAOMING ZHANG ${ }^{1}$, WUGUANG LIU ${ }^{1}$, ZEXUAN YANG ${ }^{1}$, RUI LIANG ${ }^{2,3}$ and LIMING WANG ${ }^{1}$ \\ ${ }^{1}$ Department of General Surgery, Division of Hepatobiliary and Pancreatic Surgery, The Second Affiliated Hospital of \\ Dalian Medical University, Dalian, Liaoning 116044; ${ }^{2}$ Department of General Surgery, Shenzhen University General \\ Hospital/Shenzhen University Clinical Medical Academy, Shenzhen, Guangdong 518000; ${ }^{3}$ Carson International \\ Cancer Research Centre, Shenzhen University School of Medicine, Shenzhen, Guangdong 518055, P.R. China
}

Received January 7, 2020; Accepted July 14, 2020

DOI: $10.3892 / \mathrm{ol} .2020 .11988$

\begin{abstract}
Homeodomain-containing gene 10 (HOXC10) is associated with the progression of a variety of different types of human cancer; however, the role of HOXC10 in liver cancer is not completely understood. The present study aimed to investigate the mechanisms underlying the effects of HOXC10 on liver cancer tumorigenesis. Quantitative PCR and western blotting were used to detect the expression patterns of HOXC10 in cancer and adjacent healthy tissues. EdU, Cell Counting Kit- 8 and colony formation assays were used to determine the functions of HOXC10 in liver cancer cell lines. ENCORI, TargetScan and miRTarBase were used to identify microRNAs that target HOXC10. The verification of the interaction between HOXC10 and microRNA-221 was determined by a luciferase assay. Compared with adjacent non-cancerous tissues, the expression of HOXC10 was markedly decreased in liver cancer tissues. A HOXC10 small interfering (si)RNA significantly attenuated HOXC10 expression at the mRNA and protein levels, and enhanced cell proliferation compared with the siRNA-negative control group. In addition, the luciferase
\end{abstract}

Correspondence to: Professor Liming Wang, Department of General Surgery, Division of Hepatobiliary and Pancreatic Surgery, The Second Affiliated Hospital of Dalian Medical University, 467 Zhongshan Road, Dalian, Liaoning 116044, P.R. China

E-mail: wangbcc259@163.com

Dr Rui Liang, Department of General Surgery, Shenzhen University General Hospital/Shenzhen University Clinical Medical Academy, 1089 Xueyuan Avenue, Shenzhen, Guangdong 518000, P.R. China E-mail: 1r9311038@yahoo.com

*Contributed equally

Key words: homeodomain-containing gene 10, liver cancer, proliferation, microRNA-221, mitogen-activated protein kinase signaling pathway reporter assay indicated that microRNA-221 directly bound to the 3'-untranslated region of $\mathrm{HOXC10}$, and interfered with the inhibitory effect of $\mathrm{HOXC10}$ on proliferation. In addition, HOXC10 knockdown elevated the expression levels of mitogen-activated protein kinase signaling pathway markers compared with the siRNA-negative control group. Therefore, the results of the present study may aid with the development of novel therapeutic regimens and diagnostic markers of liver cancer.

\section{Introduction}

Liver cancer is one of the leading causes of cancer-related death worldwide (1). Despite developments in liver cancer treatment, with improved surgical techniques and interventional therapies, the 5-year survival rate remains poor and the mortality rate has increased significantly over the past 20 years $(2,3)$. Unlike other organs, the liver displays some potential to regenerate, but the proliferation rate of liver cancer cells is higher compared with that of normal liver cells due to several deregulated signaling pathways during cancer, such as PTEN, PI3K and AKT (4). Such deregulation occurs throughout all stages of tumor development, which leads to inconsistent and ineffective diagnosis and treatment of liver cancer (5). To improve the diagnosis and monitor the progression of liver cancer in patients, the development of more accurate and stable biomarkers is required.

The HOX gene family is highly conserved across species; according to the distribution of the HOX gene family in different chromosomes, four subgroups with genomic clusters of A-D were established (6,7). Primarily, the HOX gene family drives normal cellular differentiation and morphogenesis in embryonic stages, and regulates normal tissue morphology in adults (8). HOX genes have been reported to be abnormally expressed in leukemia, breast and gastric cancer $(9,10)$. In addition, it has been reported that the HOX gene family alters the occurrence and development of malignant tumors (11), indicating that the HOX gene family may serve as potential biomarkers of cancer. HOXC10, a member of the HOX gene 
family, promotes tumor cell migration and invasion in multiple forms of cancer, including glioma (12), lung cancer (13), breast cancer $(14,15)$, thyroid cancer $(16)$, gastric cancer $(17,18)$ and osteosarcoma (19). However, the role of HOXC10 in liver cancer is not completely understood. The present study aimed to investigate the role of HOXC10 in liver cancer, and identify the molecular mechanisms by which HOXC10 alters the development and progression of liver cancer.

\section{Materials and methods}

Patients and tissue sample. A total of 40 paired specimens were retrospectively collected from patients with primary liver cancer who received treatment at the Second Affiliated Hospital of Dalian Medical University between January 2016 and December 2019. Samples were collected intraoperatively and immediately snap-frozen with liquid nitrogen. Adjacent healthy tissues were obtained $\geq 5 \mathrm{~cm}$ from the edge of the tumor. All specimens were pathologically confirmed as liver cancer. Comprehensive reports of all clinical and pathological examinations were provided for each patient. The clinical characteristics of the patients are provided in Table I. The present study was approved by the Ethics Committee of the Second Affiliated Hospital of Dalian Medical University (approval no. 2019.087). Written informed consent was obtained from all the participants. Patients with a history of tumor resection surgery, chemotherapy, radiotherapy, second primary tumors or other malignant diseases were excluded from the present study.

Cell culture and cell lines. A total of seven liver cancer cell lines (Huh7, MHCC97-H, MHCC97-L, HepG2, Hep3B, Snu449 and PLC/PRF/5) were obtained from American Type Culture Collection and authenticated via STR profiling. Cells were cultured in RPMI-1640 (cat. no. SH30027.01; HyClone; Cytiva), DMEM (cat. no. 11965118; Thermo Fisher Scientific, Inc.) or EMEM (cat. no. 670086; Thermo Fisher Scientific, Inc.) supplemented with $1 \%$ antibiotics $(100 \mathrm{mg} / \mathrm{ml}$ streptomycin and $100 \mathrm{U} / \mathrm{ml}$ penicillin) and 10\% FBS (cat. no. 26140079; Gibco; Thermo Fisher Scientific, Inc.) at $37^{\circ} \mathrm{C}$ with $5 \% \mathrm{CO}_{2}$.

Western blotting. Total protein was extracted from liver cancer cells and tissues using lysis RIPA buffer (cat. no. P0013B; Beyotime Institute of Biotechnology) containing phosphatase and protease inhibitors. Total protein was quantified using a standard bicinchoninic acid protein assay kit (cat. no. 23225; Thermo Fisher Scientific, Inc.). Proteins were diluted in 5X loading buffer (cat. no. P0015L; Beyotime Institute of Biotechnology) and subsequently, proteins (15 $\mu \mathrm{g}$ per lane) were separated via $10 \%$ SDS-PAGE and transferred to PVDF membranes at $250 \mathrm{~mA}$ for $2 \mathrm{~h}$. The membranes were blocked with $5 \%$ skim milk at room temperature for $1 \mathrm{~h}$. Subsequently, the membranes were incubated at $4^{\circ} \mathrm{C}$ for $16 \mathrm{~h}$ with primary antibodies targeted against: HOXC10 (cat. no. SC-517164; 1:1,000; Santa Cruz Biotechnology, Inc.), GAPDH (cat. no. 5174S; 1:1,000; Cell Signaling Technology, Inc.), JNK (cat. no. 9252S; 1:1,000; Cell Signaling Technology, Inc.), phosphorylated (p)-JNK (cat. no. 9255S; 1:2,000; Cell Signaling Technology, Inc.), ERK (cat. no. 4695S; 1:1,000; Cell Signaling Technology, Inc.) and p-ERK (cat. no. 4370S; 1:2,000;
Cell Signaling Technology, Inc.). Following primary incubation, the membranes were incubated with anti-mouse IgG and anti-rabbit IgG secondary antibodies (cat. nos. 7076S and 7074S; 1:2,000; Cell Signaling Technology, Inc.) for $1 \mathrm{~h}$ at room temperature. Protein bands were visualized using an ECL kit (cat. no. P0018FS; Beyotime Institute of Biotechnology). GAPDH was used as the loading control. ImageJ version 1.8.0 112 bundled with 64-bit Java (National Institutes of Health) was used for densitometry analysis.

Reverse transcription-quantitative PCR (RT-qPCR). Total RNA was extracted from cells and tissues using TRIzol ${ }^{\circledR}$ reagent (Invitrogen; Thermo Fisher Scientific, Inc.). Total RNA was diluted in RNase-free DNase solution and reverse transcribed into cDNA using PrimeScript RT Reagent Kit with gDNA Eraser (cat. no. RR047A; Takara Bio, Inc.) on a TP350 thermal cycler (Takara Bio, Inc.). The following temperature protocol was used for reverse transcription: $42^{\circ} \mathrm{C}$ for $2 \mathrm{~min}$ for gDNA Eraser; followed by first strand cDNA synthesis at $37^{\circ} \mathrm{C}$ for $15 \mathrm{~min}$ and $85^{\circ} \mathrm{C}$ for $5 \mathrm{sec}$. Subsequently, qPCR was performed using the Mx3005P real-time PCR system (Agilent Technologies, Inc.) and SYBR Premix Ex Taq kit (Applied Biosystems; Thermo Fisher Scientific, Inc.). The following thermocycling conditions were used for qPCR: Initial denaturation at $95^{\circ} \mathrm{C}$ for $30 \mathrm{sec}$; followed by 40 cycles of $95^{\circ} \mathrm{C}$ for $5 \mathrm{sec}$ and $72^{\circ} \mathrm{C}$ for $30 \mathrm{sec}$, and a final extension at $72^{\circ} \mathrm{C}$ for $30 \mathrm{sec}$. The following primers were used for qPCR: HOXC10 forward, 5'-ACATCTGGAATCGCCTCAGC-3' and reverse, 5'-GGCTCTGCTCCGTCTTGATT-3'; and $\beta$-actin (ACTB) forward, 5'-ATGTGGCCGAGGACTTTGATT-3' and reverse, 5'-AGTGGGGTGGCTTTTAGGATG-3'. HOXC10 mRNA expression levels were quantified using the $2^{-\Delta \Delta \mathrm{Cq}}$ method (20) and normalized to the internal reference gene ACTB.

To assess microRNA (miRNA/miR) expression levels, total RNA $(1 \mu \mathrm{g})$ was reverse transcribed using an ImProm-II ${ }^{\mathrm{TM}}$ Reverse Transcription kit (Promega Corporation) according to the manufacturer's protocol. The following temperature protocol was used for reverse transcription: $25^{\circ} \mathrm{C}$ for $5 \mathrm{~min}$ for annealing, followed by extension at $42^{\circ} \mathrm{C}$ for $45 \mathrm{~min}$ and final inactivation of the reverse transcriptase at $70^{\circ} \mathrm{C}$ for $15 \mathrm{~min}$. Subsequently, qPCR was performed using a QuantStudio Dx Real-Time instrument (Thermo Fisher Scientific, Inc.) with the SYBR Premix Ex Taq Kit (Takara Bio, Inc.). The following thermocycling conditions were used for qPCR: Initial denaturation at $95^{\circ} \mathrm{C}$ for $30 \mathrm{sec}$; followed by 40 cycles of $95^{\circ} \mathrm{C}$ for $5 \mathrm{sec}$ and $72^{\circ} \mathrm{C}$ for $30 \mathrm{sec}$, and a final extension at $72^{\circ} \mathrm{C}$ for $30 \mathrm{sec}$. The following primers were used for qPCR: miR-221 forward, 5'-GGGAAGCTACATTGTCTGC-3' and reverse, 5'-CAGTGCGTGTCGTGGAGT-3'; and U6 forward, 5'-GCT TCGGCAGCACATATACTAAAAT-3' and reverse, 5'-CGC TTCACGAATTTGCGTGTCAT-3'. miRNA expression levels were normalized to the internal reference gene U6. The relative level was calculated using the $2^{-\Delta \Delta \mathrm{Cq}}$ method.

miRNA and small interfering (si)RNA transfection. Cells in the logarithmic growth phase were washed with antibiotic- and serum-free fresh medium. A total of $5 \times 10^{4}$ cells/well in a 24 -well plate were used for transfection. HOXC10 siRNA-1 (5'-CGGGAAAGAAAGAGGGAA C-3'), HOXC10 siRNA-2 (5'-GCACACAAGACGCAACAA 
Table I. Association between HOXC10 expression and clinicopathological features of liver cancer.

\begin{tabular}{|c|c|c|c|c|}
\hline \multirow[b]{2}{*}{ Variable } & \multirow[b]{2}{*}{ All cases } & \multicolumn{2}{|c|}{ HOXC10 expression } & \multirow[b]{2}{*}{ P-value } \\
\hline & & Low & High & \\
\hline Sex & & & & 0.983 \\
\hline Male & 33 & $14(82.4 \%)$ & $19(82.6 \%)$ & \\
\hline Female & 7 & $3(17.6 \%)$ & $4(17.4 \%)$ & \\
\hline Median age & $59.39 \pm 11.313$ & $59.16 \pm 7.313$ & $59.63 \pm 8.447$ & 0.854 \\
\hline Tumor size & & & & 0.006 \\
\hline$\leq 3 \mathrm{~cm}$ & 10 & $2(8.7 \%)$ & $8(47.1 \%)$ & \\
\hline$>3 \mathrm{~cm}$ & 30 & $21(91.3 \%)$ & $9(52.9 \%)$ & \\
\hline Glisson capsule invasion & & & & 0.789 \\
\hline No & 22 & $12(54.5 \%)$ & $10(58.8 \%)$ & \\
\hline Yes & 18 & $10(45.5 \%)$ & $8(41.2 \%)$ & \\
\hline Tumor differentiation & & & & 0.767 \\
\hline Well & 2 & $1(4.2 \%)$ & $1(6.3 \%)$ & \\
\hline Poor or moderate & 38 & $23(95.8 \%)$ & $15(93.8 \%)$ & \\
\hline Satellite nodules & & & & 0.327 \\
\hline No & 20 & $6(40.0 \%)$ & $14(56.0 \%)$ & \\
\hline Yes & 20 & $9(60.0 \%)$ & $11(44.0 \%)$ & \\
\hline Lymphatic metastasis & & & & 0.001 \\
\hline No & 25 & $5(31.3 \%)$ & $20(83.3 \%)$ & \\
\hline Yes & 15 & $7(68.8 \%)$ & $8(16.7 \%)$ & \\
\hline Distant metastasis & & & & 0.266 \\
\hline No & 32 & $13(72.2 \%)$ & $19(86.4 \%)$ & \\
\hline Yes & 8 & $5(27.8 \%)$ & $3(13.6 \%)$ & \\
\hline TNM stage & & & & 0.005 \\
\hline I and II & 18 & $5(23.8 \%)$ & $13(68.4 \%)$ & \\
\hline III and IV & 22 & $16(76.2 \%)$ & $6(31.6 \%)$ & \\
\hline
\end{tabular}

HOXC10, homeodomain-containing gene 10; TNM, Tumor-Node-Metastasis.

A-3'), siRNA-negative control (NC; sequence not available; cat. no. siB06525141910-1-5), miR-221 mimic (5'-CUUUGG GUCGUCUGUUACAUCGA-3'; cat. no. miR10000890-1-5), miR-mimic-NC (cat. no. miR1N0000001-1-5), miR-221 inhibitor (5'-UCGAUGUAACAGACGACCCAAAG-3'; cat. no. miR21335173141-1-5) and miR-221-NC (sequence not available; cat. no. miR2N0000001-1-5) were purchased from Guangzhou RiboBio Co., Ltd.. Cells were transfected with $20 \mathrm{nM}$ siRNA, miR mimic, miR inhibitor or corresponding negative controls using Lipofectamine ${ }^{\circledR} 2000$ (Invitrogen; Thermo Fisher Scientific, Inc.) and incubated at $37^{\circ} \mathrm{C}$ with $5 \% \mathrm{CO}_{2}$. After $24 \mathrm{~h}$, the medium was replaced. Transfection efficiency was assessed via reverse transcription-quantitative PCR.

Plasmid transfection. The pcDNA3.1-HOXC10 overexpression vector was based on the pcDNA3.1 plasmid (pcDNA3.1-HA; gifted by Dr Oskar Laur (Emory School of Medicine, Emory University, Atlanta, GA, USA); Addgene plasmid no. 128034; n2t.net/addgene:128034; RRID: Addgene_128034). HOXC10 cDNA was amplified from Huh7 cells with initial denaturation at $98^{\circ} \mathrm{C}$ for $30 \mathrm{sec}$, followed by 35 cycles of $98^{\circ} \mathrm{C}$ for $10 \mathrm{sec}$, $58^{\circ} \mathrm{C}$ for $30 \mathrm{sec}$ and $72^{\circ} \mathrm{C}$ for $30 \mathrm{sec}$, and a final extension at $72^{\circ} \mathrm{C}$ for $2 \mathrm{~min}$, using Q5 High-Fidelity 2X Master Mix (New England Biolabs) and the following primers: Forward, 5'-TAA AGCTTATGACATGCCCTCGCAATG-3' and reverse, 5'-TAGAATTCCCTAATAAATTTCCAGAATCATAAT CCTCAAA-3'. PCR products were digested with HindIII and EcoRI, and cloned into pcDNA3.1 vectors digested with the same enzymes. A total of $1 \times 10^{5}$ Snu 449 and PLC cells/well in a 12-well plate cells were transfected with $2 \mu \mathrm{g}$ pcDNA3.1 vector using Lipofectamine ${ }^{\circledR} 2000$ Transfection Reagent (cat. no. 11668019; Invitrogen; Thermo Fisher Scientific, Inc.) according to the manufacturer's protocol. At $48 \mathrm{~h}$ post-transfection, cells were used for subsequent experiments.

Cell counting Kit-8 (CCK-8) assay. At 24 h post-transfection, the CCK-8 assay (cat. no. CK04-05; Dojindo Molecular Technologies, Inc.) was performed to assess Huh7 and $97 \mathrm{H}$ cell viability according to the manufacturer's protocol. Briefly, cells $\left(1 \times 10^{2}\right.$ cells/well) were incubated in 96 -well plates with $10 \mu \mathrm{l} \mathrm{CCK}-8$ reagent. At $0,12,24,36,48$ and $72 \mathrm{~h}$, cell viability 


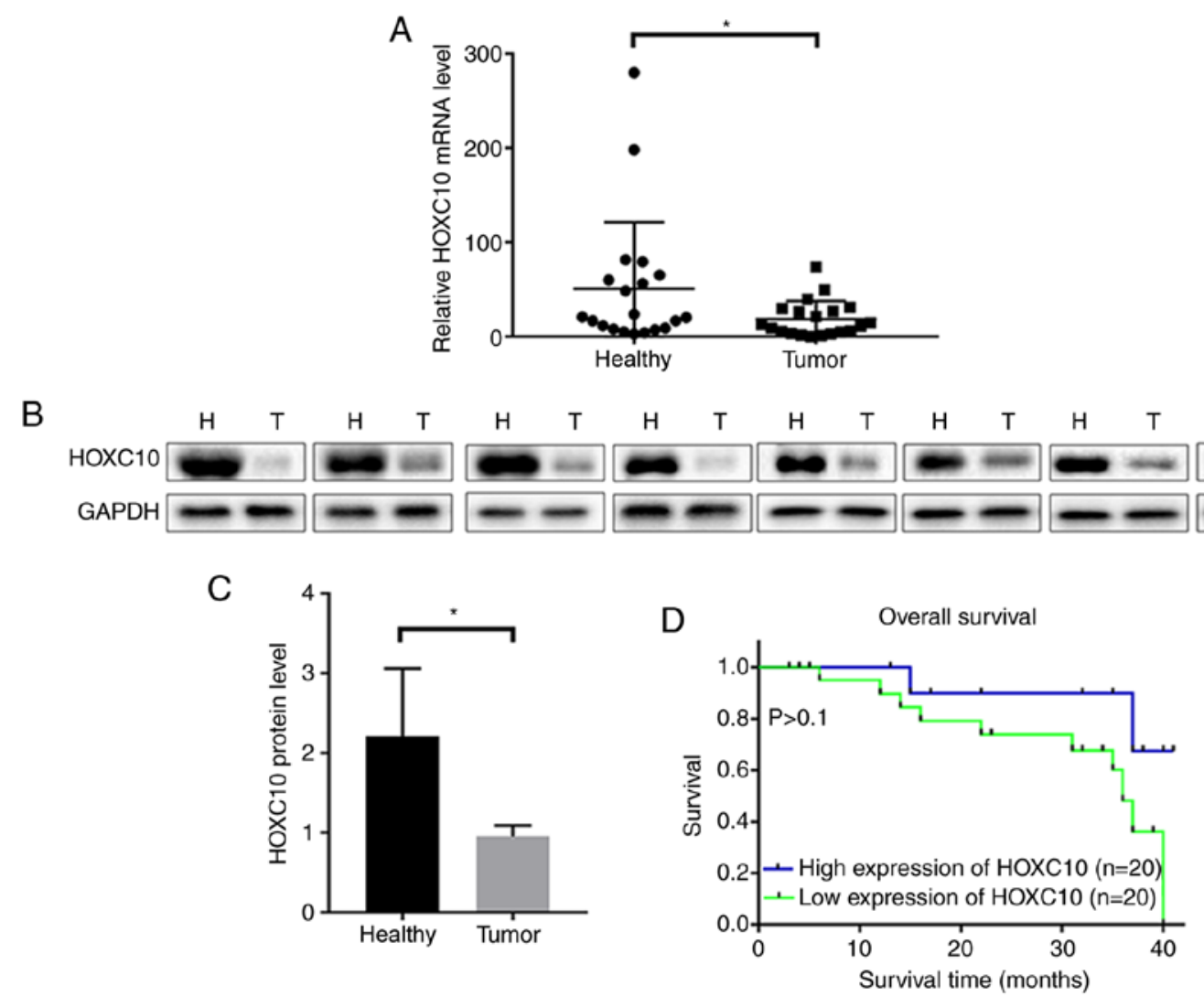

Figure 1. Clinical relevance and expression pattern of HOXC10 in human liver cancer. (A) HOXC10 mRNA expression levels in 40 paired liver cancer and adjacent non-cancerous tissues. HOXC10 protein expression levels in 40 paired liver cancer and adjacent non-cancerous tissues were (B) determined by western blotting and (C) semi-quantified. (D) Patients were divided into two groups according to the mean value of HOXC10 mRNA expression. Survival analysis was performed using the Kaplan-Meier method and survival curves were compared using the log-rank test. ${ }^{*} \mathrm{P}<0.05$. HOXC10, homeodomain-containing gene 10 ; $\mathrm{N}$, normal; $\mathrm{T}$, tumor.

was measured at a wavelength of $450 \mathrm{~nm}$ using a microplate reader.

Colony formation assay. Huh7, 97H, Snu449 and PLC cells $\left(2 \times 10^{2}\right.$ cells/well) were cultured in $60-\mathrm{mm}$ wells with $5 \mathrm{ml}$ medium for $24 \mathrm{~h}$ at $37^{\circ} \mathrm{C}$ with $5 \% \mathrm{CO}_{2}$. Once a visible clone was formed, the colonies were stained using $1 \%$ crystal violet solution for $10 \mathrm{~min}$. Following washing with PBS, the colonies were dried, examined and counted. Clusters of $\geq 50$ cells (size, 0.3-1.0 mm) were considered as colonies. Images were obtained using an HP scanner (Laserjet 100 color MFP; Hewlett-Packard).

Cell proliferation assay. Cell proliferation was assessed using a Cell-Light EdU DNA Cell Proliferation kit (cat. no. C10310-1; Guangzhou RiboBio Co., Ltd.) according to the manufacturer's protocol. Huh7, 97H, Snu449 and PLC cells were examined using a fluorescence microscope (x20 magnification), and cell proliferation was determined as the ratio of EdU-positive cells to Hoechst-positive cells (21).

Luciferase reporter assay. To investigate the regulation of HOXC10 expression by miRNA, a luciferase reporter assay was performed. To identify the potential regulatory miRNAs of HOXC10, ENCORI (http://starbase.sysu.edu.cn/), TargetScan (http://www.targetscan.org/mamm_31/) and miRTarBase (http://mirtarbase.mbc.nctu.edu.tw/php/index.php) databases were used. Mutant (MUT)-HOXC10 or wild-type (WT)-HOXC10 were amplified using Q5 High-Fidelity 2X Master Mix (New England Biolabs) with cDNA from Huh7 and the following primers: MUT-HOXC10 forward, 5'-GCG ACgettttttGGCAAAGACCTCAGACTCTCCTT-3' and reverse, 5'-TTGCCaaaaaagcGTCGCATTGCATTTATA CTCAGGG-3'; WT-HOXC10 forward, 5'-aattctaggcgatcgctc gagATCGGATCCGGGAACTGACC-3' and reverse, 5'-aaa cgaattccegggetcgagGAACCACAGGTCCCTTGGAAG-3'.

The amplification was performed with initial denaturation at $98^{\circ} \mathrm{C}$ for $30 \mathrm{sec}$, followed by 35 cycles of $98^{\circ} \mathrm{C}$ for $10 \mathrm{sec}$, $58^{\circ} \mathrm{C}$ for $30 \mathrm{sec}$ and $72^{\circ} \mathrm{C}$ for $30 \mathrm{sec}$, and final extension at $72^{\circ} \mathrm{C}$ for $2 \mathrm{~min}$. The amplified fragments were linked into the psi-CHECK2 vector (Promega Beijing Biotech Co., Ltd.). For the luciferase reporter assay, at 70-80\% confluence, Huh7 cells were transfected with $0.5 \mu \mathrm{g}$ MUT HOXC10-3'-untranslated region (UTR; MUT-HOXC10) or WT HOXC10-3'UTR using Lipofectamine ${ }^{\circledR} 2000$ (Invitrogen; Thermo Fisher Scientific, Inc.). The concentration of mimic-221, inhibitor-221, mimic-NC and inhibitor-NC used for co-transfection was $50 \mathrm{nM}$. At $48 \mathrm{~h}$ post-transfection, luciferase activity was assessed using the Dual-Luciferase Activity Assay system (Promega Corporation).

Statistical analysis. Statistical analyses were conducted using GraphPad Prism (version 7; GraphPad Software, Inc.) and SPSS (version 17.0; SPSS, Inc.) software. Comparisons 


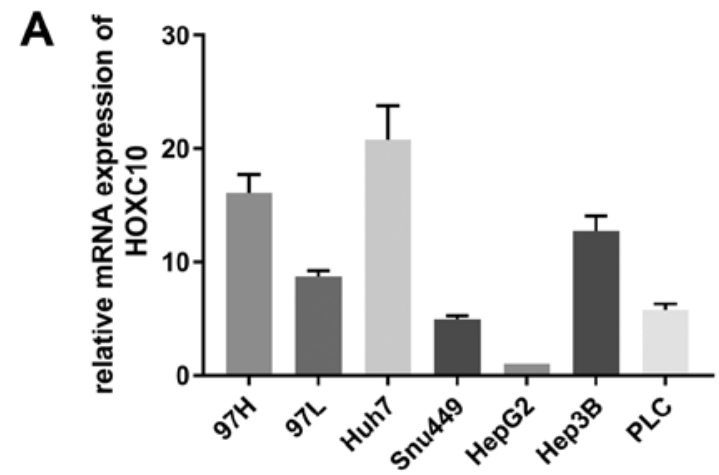

\section{B 97н}

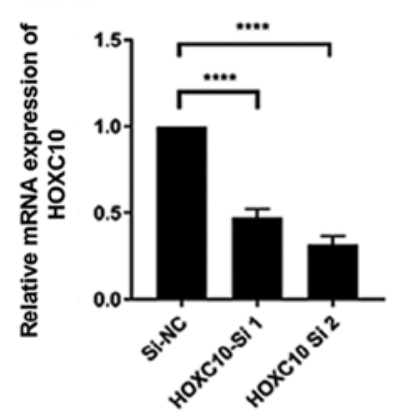

C Snu449

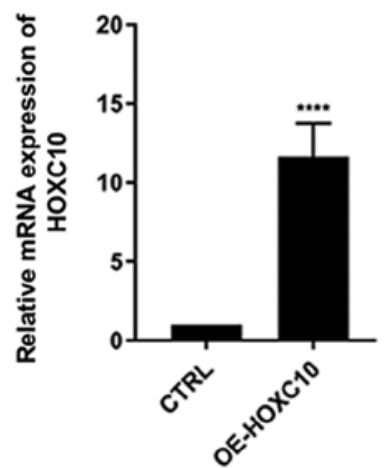

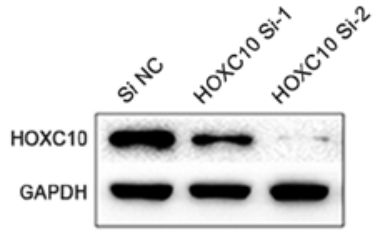

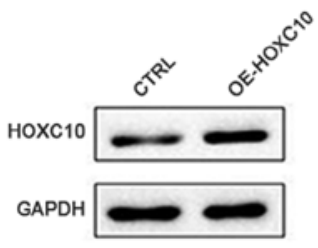

Huh7

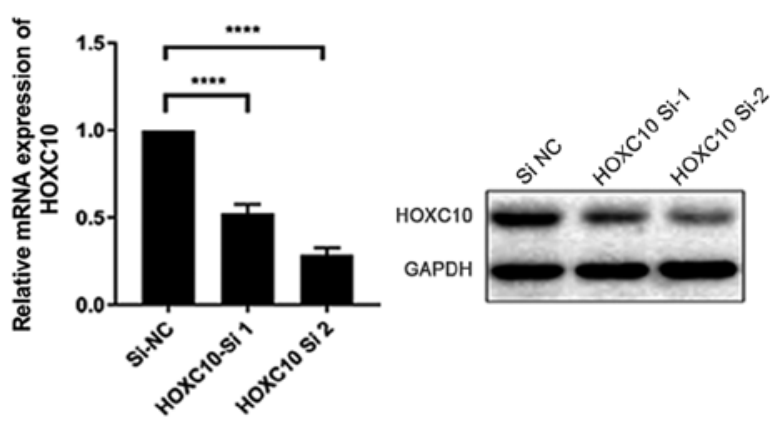

PLC

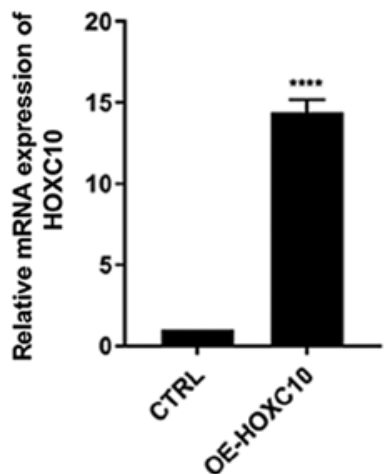

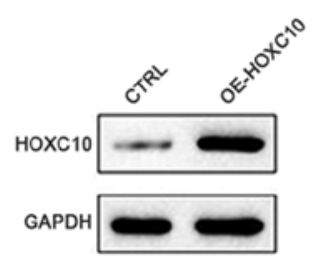

Figure 2. HOXC10 knockdown and overexpression in human liver cancer cells. (A) HOXC10 mRNA expression levels in liver cancer cell lines. (B) si-HOXC10 transfection efficiency in Huh7 and 97H cells. (C) pcDNA3.1-HOXC10 transfection efficiency in Snu449 and PLC cells. ${ }^{* * * * *}$ P $<0.0001$. HOXC10, homeodomain-containing gene 10; si, small interfering RNA; NC, negative control; CTRL, control.

among multiple groups were analyzed using one-way ANOVA followed by Tukey's post hoc test. Comparisons between two groups were analyzed using the paired or unpaired Student's t-test. Data presented as $n(\%)$ were analyzed using the $\chi^{2}$ test and data presented as the mean \pm interquartile range were analyzed using Student's t-test. The prognostic potential of HOXC10 was determined using Kaplan-Meier survival curves, which were compared using log-rank tests. Data are presented as the mean \pm standard deviation. $\mathrm{P}<0.05$ was considered to indicate a statistically significant difference.

\section{Results}

HOXC10 is downregulated in liver cancer tissues. The expression levels of HOXC10 in 40 paired liver cancer and adjacent non-cancerous tissues were assessed via RT-qPCR and western blotting. HOXC10 mRNA (Fig. 1A) and protein
(Fig. 1B and C) expression levels were significantly downregulated in liver cancer tissues compared with adjacent non-cancerous tissues.

The clinical characteristics of the liver cancer tissues are presented in Table I. The cases were divided into two groups (low expression and high expression) according to the median of the mRNA expression level of HOXC10. Patients in the low HOXC10 expression group displayed increased tumor size, more severe lymphatic metastasis and worse TNM stage (22) compared with patients with high HOXC10 expression, suggesting that low HOXC10 expression may be associated with increased tumor aggression. Although not statistically significant, the Kaplan-Meier survival curves indicated that low HOXC10 expression levels may correlate with poor survival in patients with liver cancer (Fig. 1D). Further studies with larger sample sizes are required to validate the results of the present study. 
A
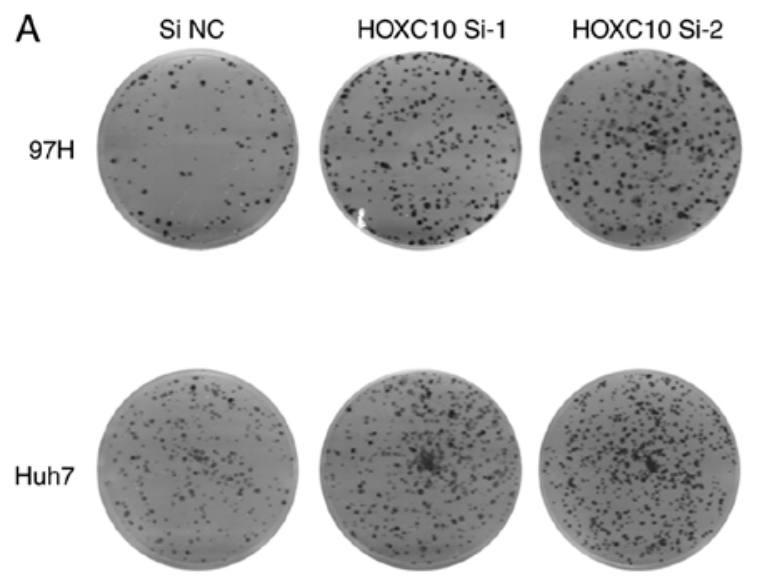

C

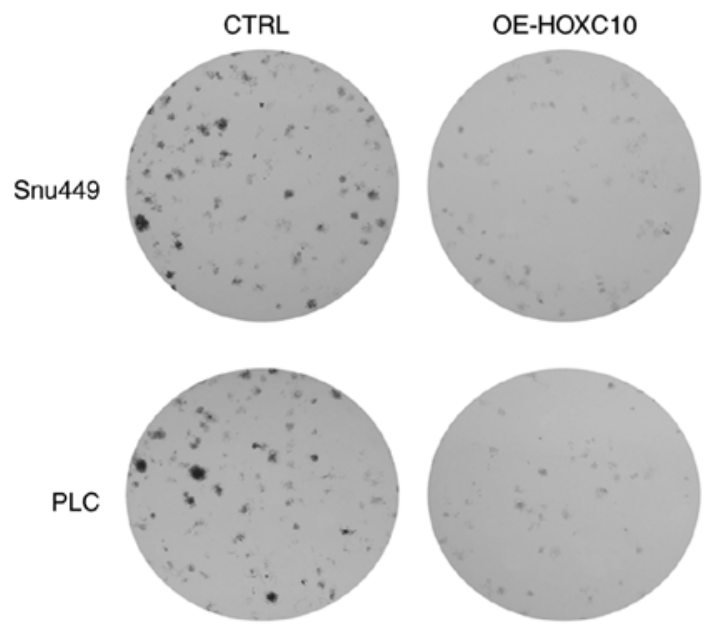

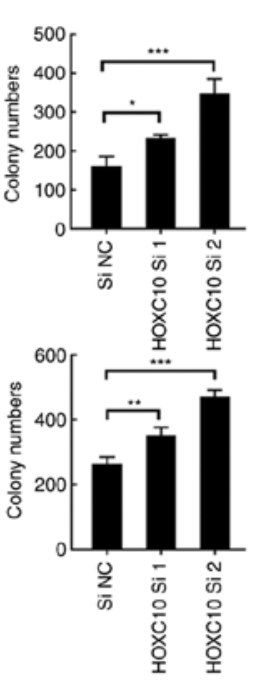

B
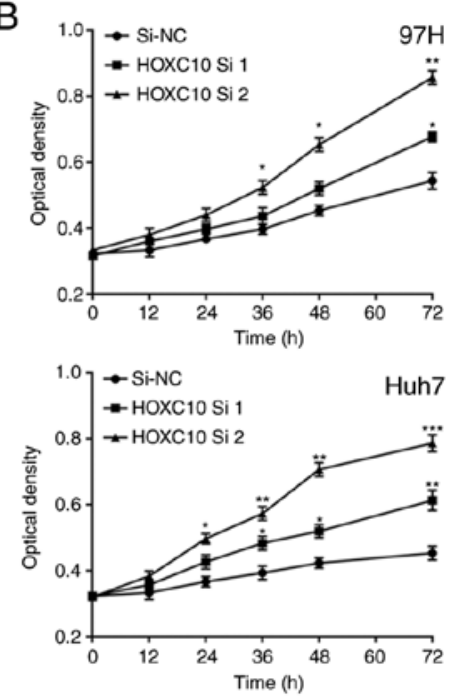

D
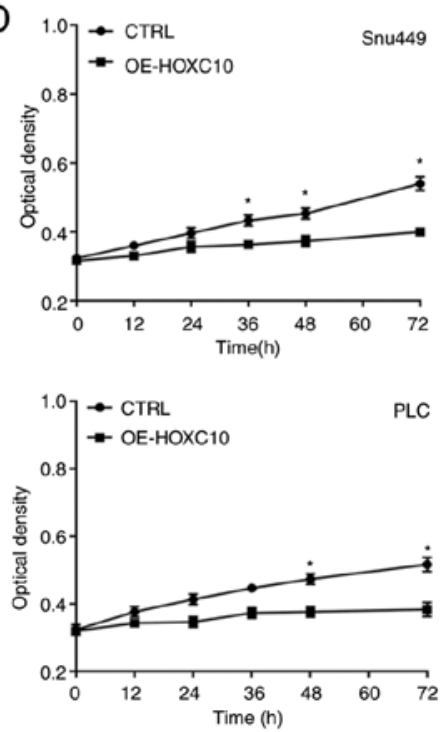

Figure 3. HOXC10 suppresses liver cancer cell proliferation. Effect of si-HOXC10 on (A) colony formation and (B) cell viability. Effect of pcDNA3.1-HOXC10 on (C) colony formation and (D) cell viability. ${ }^{*} \mathrm{P}<0.05,{ }^{* *} \mathrm{P}<0.01$ and ${ }^{* * *} \mathrm{P}<0.001$. HOXC10, homeodomain-containing gene 10; si, small interfering RNA; NC, negative control; CTRL, control; OE, overexpression.

HOXC10 suppresses liver cancer cell viability. HOXC10 mRNA expression in seven different liver cancer cell lines was assessed (Fig. 2A). The results indicated that HOXC10 mRNA expression levels were notably increased in Huh7 and 97H cells compared with other liver cancer cell lines. Therefore, Huh7 and $97 \mathrm{H}$ cell lines were used for loss-of-function experiments, whereas Snu449 and PLC cell lines were used for gain-of-function experiments. HOXC10 knockdown and overexpression efficiency were assessed via RT-qPCR and western blotting. HOXC10 siRNA notably decreased HOXC10 mRNA and protein expression levels in $\mathrm{Huh} 7$ and $97 \mathrm{H}$ cells compared with the si-NC group (Fig. 2B). By contrast, mRNA and protein expression levels of $\mathrm{HOXC10}$ were notably increased in the pcDNA3.1-HOXC10 group compared with the empty vector group (Fig. 2C).

Subsequently, colony formation and CCK- 8 assays were performed to evaluate the effect of $\mathrm{HOXC10}$ on liver cancer cell viability. Compared with the si-NC group, HOXC10 knockdown significantly increased colony formation and cell viability (Fig. 3A and B). By contrast, compared with the empty vector group, HOXC10 overexpression significantly decreased colony formation and cell viability (Fig. 3C and D).

Collectively, the results indicated that HOXC10 suppressed liver cancer cell viability.

miR-221 downregulates HOXC10 expression in liver cancer by directly targeting its 3'-UTR. The aforementioned results of the present study demonstrated that $\mathrm{HOXC10}$ expression was altered in liver cancer tissues and regulated tumor cell proliferation; therefore, it was further investigated how HOXC10 expression may be regulated in liver cancer. miR-221 was identified as a regulatory miRNA of $\mathrm{HOXC10}$ by bioinformatics analysis as the seed region of $\mathrm{HOXC10}$ was highly complementary to miR-221. The results indicated that miR-221 may downregulate HOXC10 in liver cancer; therefore, miR-221 was selected for further analysis in the present study. 
A

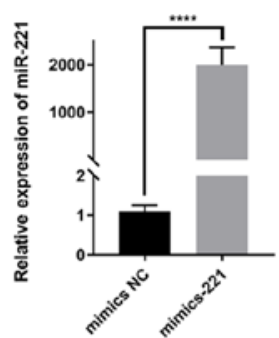

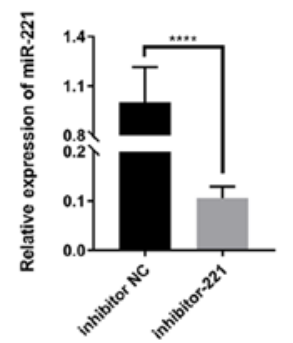
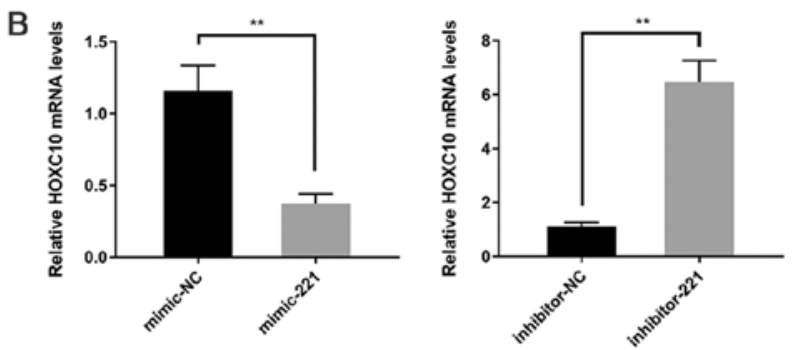

C WT HOXC10 3'UTR 5'...UGUUGUAUCUUUUGCAUGUAGCU... 3

||||||

hsa-mir-221-3p 5' CUUUGGGUGUCUGUUACAUCGA $33^{\prime}$

MUT HOXC10 3'UTR 5'...UGUUGUAUCUUUUGCCGUGCUAU... 3'
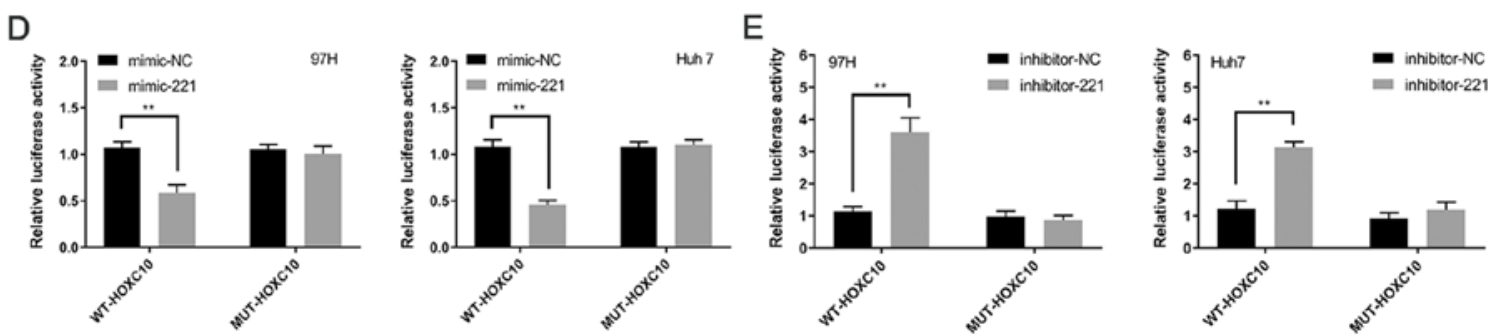

Figure 4. miR-221 directly targets the 3'UTR of HOXC10. (A) Transfection efficiency of miR-221 mimic and miR-221 inhibitor. (B) HOXC10 mRNA expression levels in miR-221 mimic- and miR-221 inhibitor-transfected Huh7 cells. (C) The binding site between miR-221 and HOXC10. (D) The luciferase reporter assay in $97 \mathrm{H}$ and Huh7 cells transfected with WT-HOXC10 or MUT-HOXC10 and miR-221 mimic or mimic-NC. (E) The luciferase reporter assay in 97H and Huh7 cells transfected with WT-HOXC10 or MUT-HOXC10 and miR-221 inhibitor or inhibitor-NC. ${ }^{* *} \mathrm{P}<0.01$ and ${ }^{* * * * *} \mathrm{P}<0.001$. miR, microRNA; 3 'UTR, 3'-untranslated region; HOXC10, homeodomain-containing gene 10; WT, wild-type; MUT, mutant; NC, negative control.

To confirm that miR-221 downregulated HOXC10, miR-221 mimic, miR-221 inhibitor and the corresponding NCs were transfected into Huh7 cells. miR-221 knockdown and overexpression efficiency were assessed via RT-qPCR (Fig. 4A). Subsequently, HOXC10 expression levels were detected via RT-qPCR. HOXC10 expression levels were significantly decreased in miR-221 mimic-transfected Huh7 cells compared with miR-221 inhibitor- and negative control-transfected Huh7 cells (Fig. 4B). In addition, based on the seed sequence predicted by the databases, luciferase plasmids containing the WT or MUT 3'UTR of HOXC10 were constructed (Fig. 4C). miR-221 mimic significantly decreased the luciferase activity of WT-HOXC10-3'UTR compared with mimic-NC, whereas the luciferase activity of MUT-HOXC10-3'UTR was not significantly altered by miR-221 mimic compared with mimic-NC (Fig. 4D). miR-221 inhibitor significantly increased the luciferase activity of WT-HOXC10-3'UTR compared with inhibitor-NC, whereas the luciferase activity of MUT-HOXC10-3'UTR was not significantly altered by miR-221 inhibitor compared with inhibitor-NC (Fig. 4E). The results indicated that miR-221 directly interacted with the 3'UTR of HOXC10 mRNA and downregulated HOXC10 expression.

miR-221 interferes with the inhibitory effect of HOXC10 on proliferation. Based on the interaction between miR-221 and HOXC10, whether miR-221 could affect the functionality of HOXC10 in liver cancer was investigated. Therefore, Huh7 cells were transfected with HOXC10 siRNA in the presence of miR-221 inhibitor or the corresponding NC. HOXC10 knockdown significantly increased colony formation compared with the si-NC+ inhibitor-NC group. The colony formation assay also indicated that miR-221 inhibitor partially reversed HOXC10 siRNA-induced increases in colony formation (Fig. 5A). The EdU assay results indicated that HOXC10 knockdown significantly increased cell proliferation compared with the si-NC and inhibitor-NC group. Similarly, miR-221 inhibitor partially reversed HOXC10 siRNA-induced cell proliferation (Fig. 5B). The results suggested that miR-221 interfered with the inhibitory effect of $\mathrm{HOXC10}$ on liver cancer cell proliferation.

HOXC10 is associated with the mitogen-activated protein kinase (MAPK) signaling pathway. Abnormal activation or overactivation of MAPK modifies the malignant transformation and evolution of cells (23). To investigate whether HOXC10 knockdown affected MAPK signaling in liver cancer, the expression levels of MAPK marker proteins were measured, as the phosphorylation levels of ERK and JNK partly indicate activation of the MAPK signaling pathway. p-ERK and p-JNK expression levels were markedly elevated in the si-HOXC10 group compared with the si-NC group, whereas total ERK and JNK were not notably altered (Fig. 6). The results indicated that $\mathrm{Huh} 7$ and $97 \mathrm{H}$ cell proliferation may be modulated via activation of MAPK signaling in response to HOXC10.

\section{Discussion}

Despite significant progress in the past decades in the diagnosis, surgery and chemotherapy of liver cancer, there has been no significant improvement in the 5-year survival rate (1). Tumorigenesis and development are a complex regulatory network involving numerous genes $(24,25)$. The present study 
A

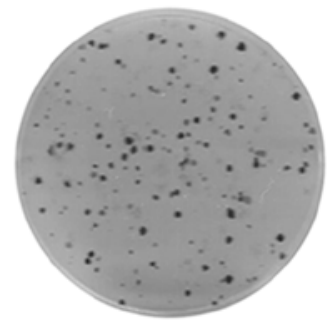

Si NC+inhibitor NC

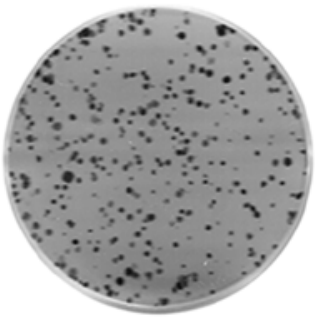

Si HOXC10+inhibitor NC

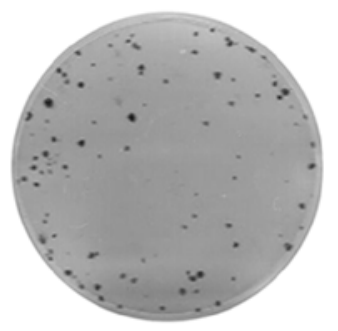

Si NC+inhibitor 221

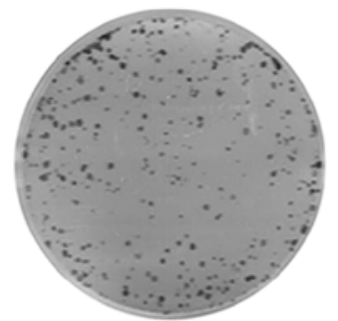

Si HOXC10+inhibitor 221

B
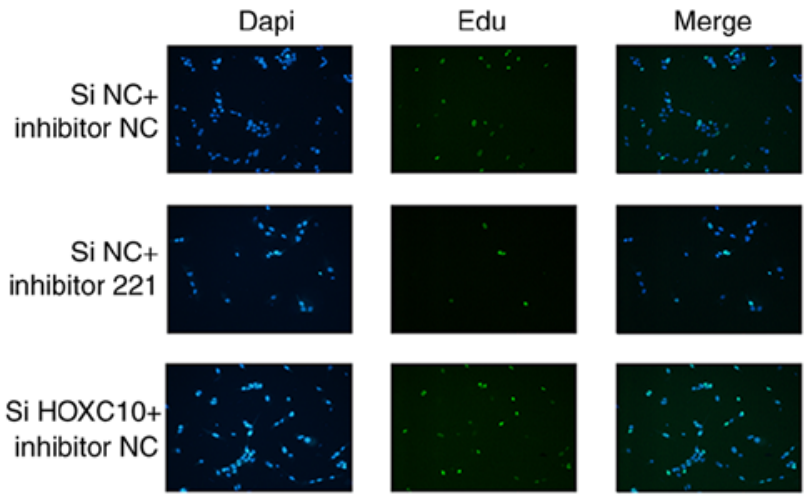

Si HOXC10+
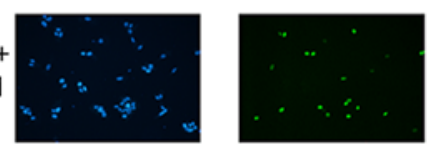
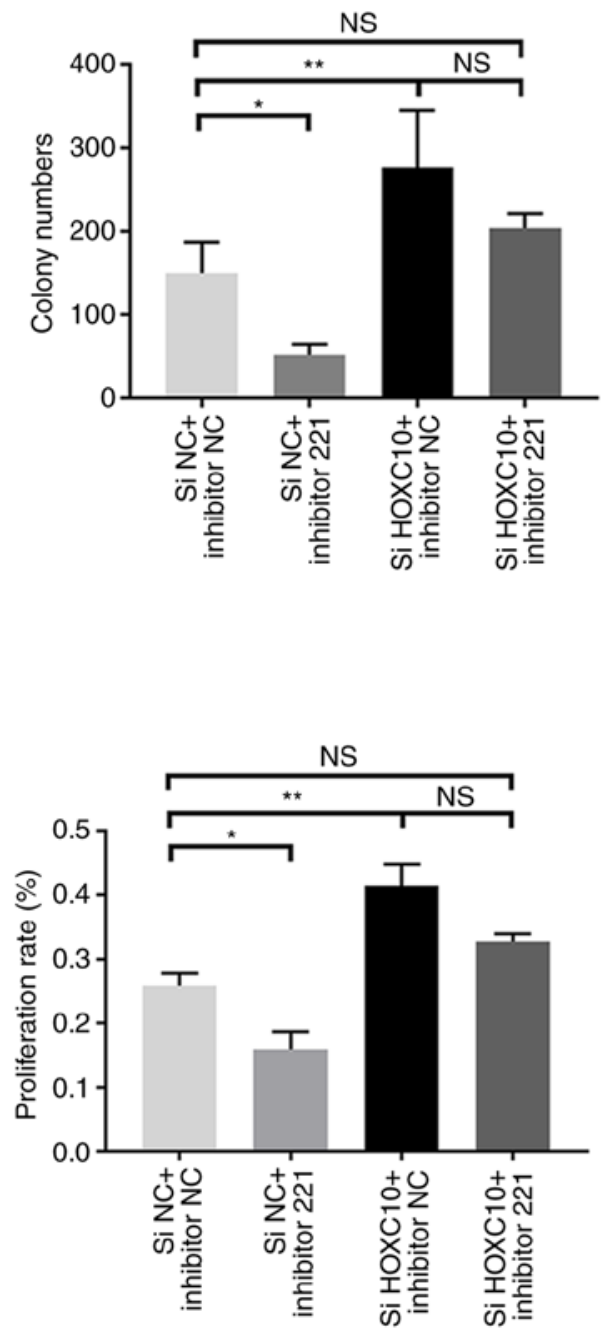

Figure 5. miR-221 interferes with the inhibitory effects of HOXC10 on cell proliferation. (A) Colony formation and (B) EdU assays indicated that miR-221 regulated cell proliferation by targeting HOXC10 in liver cancer (x20 magnification). ${ }^{*} \mathrm{P}<0.05$ and ${ }^{* *} \mathrm{P}<0.01$. miR, microRNA; HOXC10, homeodomain-containing gene 10; si, small interfering RNA; NC, negative control; NS, not significant.

explored the function of HOXC10 and its regulation in liver cancer.

First, the expression of HOXC10 in liver cancer specimens and adjacent non-cancerous tissues was assessed. HOXC10 expression was downregulated in liver cancer tissues compared with adjacent non-cancerous tissues, which was associated with worse prognosis and survival outcome. Moreover, HOXC10 overexpression and knockdown suppressed and promoted liver cancer cell proliferation, respectively. However, the upstream and downstream regulators and targets of HOXC10 have no yet been identified. Via ENCORI, TargetScan and miRTarBase, miRNA-221 was predicted to bind to the 3'UTR of HOXC10. The luciferase reporter assay indicated that miR-221 interacted with $\mathrm{HOXC10}$ to downregulate its expression. As the majority of the tissues were used in other experiments, the present study did not investigate the inverse correlation between miR-221 and HOXC10 expression in tissue samples from patients with liver cancer. In addition, the expression level in a normal liver cell line was not assessed, both of which were limitations of the present study. Rescue experiments suggested that miR-221 inhibitor suppressed liver cancer cell proliferation compared with inhibitor NC. The relationship between HOXC10 and the MAPK signaling pathway has been studied in gastric cancer (17); however, whether HOXC10 affects the MAPK signaling pathway in liver cancer is not completely understood. A significant increase in the expression levels of MAPK signaling pathway marker proteins was observed in the present study following HOXC10 knockdown compared with the si-NC group, which indicated that HOXC10 negatively affected the MAPK signaling pathway.

The HOX genes are essential regulators of the expression of genes involved in key cellular processes, differentiation and cell identity (9). Apart from their roles in tissues remodeling, the HOX gene network serves key roles in leukemogenesis and hematopoiesis (10). Numerous studies have demonstrated that the HOX gene family is closely related to the occurrence and development of various types of cancer (6-9,26). In liver cancer, HOXA13 and HOXA7 are involved in tumor proliferation, 


\section{B Huh7}
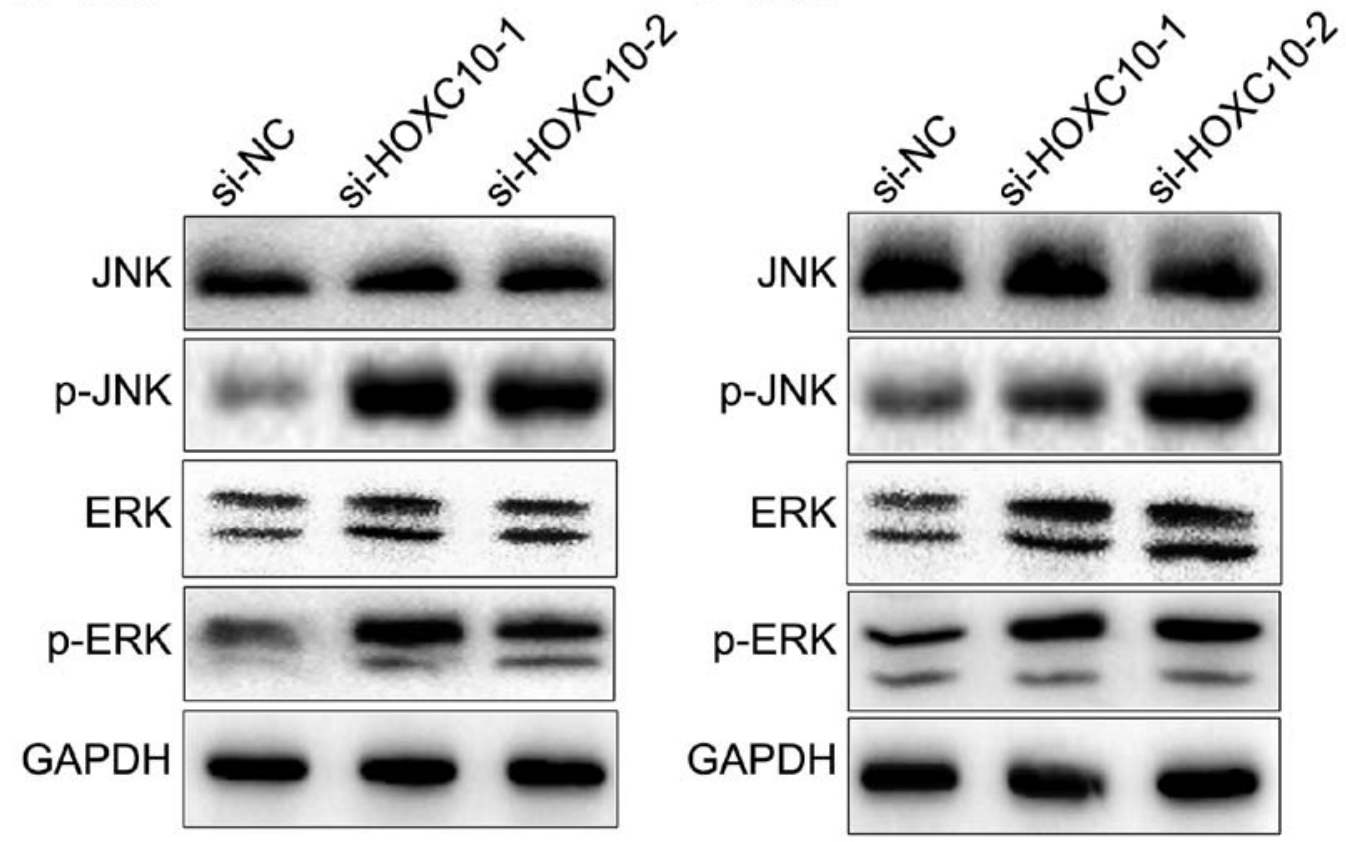

Figure 6. Effect of HOXC10 on activation of the MAPK signaling pathway. Effect of si-HOXC10 on activation of the MAPK signaling pathway in (A) 97H and (B) Huh7 cells. HOXC10, homeodomain-containing gene 10; MAPK, mitogen-activated protein kinase; si, small interfering RNA; p, phosphorylated; NC, negative control.

migration and invasion (26). Together with the results of the present study, the aforementioned studies indicated that HOXC10 and the HOX gene family may serve as biomarkers for liver cancer.

HOXC10 has been reported to be oncogenic in other tumors $(12,13)$, but in the present study, the results indicated that HOXC10 served as an antioncogene. This discrepancy regarding the function of $\mathrm{HOXC10}$ in liver cancer and other tumors may be associated with the heterogeneity of tumors, such as metastasis associated lung adenocarcinoma transcript 1 in breast cancer (27) and p53 in hepatoma carcinoma (28). In addition to this, different downstream signaling pathways can also result in different functions of the same molecule. In gastric cancer, HOXC10 serves as an oncogene via the NF-kB signaling pathway (17), whereas in breast cancer, HOXC10 supports the development of chemotherapy resistance by tuning DNA repair (14).

It has been reported that miRNAs may serve as potential tools for monitoring cancer progression, diagnosis and prognosis $(23,29)$. miRNAs are vital gene regulators that bind to partially complementary sequences at the 3'UTR of mRNAs and direct post-transcriptional modulation $(23,30)$. With the advent of miRNA expression profiles, significant efforts have been made to correlate miRNA expression with tumor development. Understanding miRNAs can reveal a more comprehensive view of the dynamic regulatory networks of cancer (31). The present study conducted a luciferase assay to assess the physical binding between miR-221 and HOXC10, and further indicated that miR-221 could effectively alter the function of HOXC10. However, the upstream regulatory molecules of HOXC10 may include other microRNAs, long non-coding RNAs and various molecules, which need to be identified in further research.
The MAPK signaling pathway controls cell apoptosis, differentiation and proliferation (32). Among its components, the roles of ERK and JNK have been extensively studied $(33,34)$. Therefore, ERK and JNK proteins were selected to assess MAPK signaling pathway activation. The results indicated that $\mathrm{HOXC10}$ knockdown increased the phosphorylation of JNK/ERK compared with the si-NC group.

In conclusion, the results of the present study suggested that HOXC10 may serve as a critical negative regulator of cell proliferation via activation of the MAPK signaling pathway. In liver cancer, HOXC10 expression was downregulated due to the suppressive effects of miR-221. Therefore, HOXC10 may serve as a novel diagnostic biomarker and therapeutic target for liver cancer.

\section{Acknowledgements}

The authors would like to thank Dr Fang Xie (Bilian Jin's Laboratory, Dalian Medical University, Dalian, China) for providing technical support and Dr Oskar Laur (Emory School of Medicine, Emory University, Atlanta, GA, USA) for providing the pcDNA3.1 plasmid.

\section{Funding}

The present study was supported by the Dalian Medical Science Research Program (grant no. 1812040).

\section{Availability of data and materials}

The datasets used and/or analyzed during the present study are available from the corresponding author on reasonable request. 


\section{Authors' contributions}

$\mathrm{CZ}, \mathrm{KM}, \mathrm{CC}$ and $\mathrm{ZF}$ designed the experiments. $\mathrm{CZ}, \mathrm{KG}$ and $\mathrm{KM}$ collected the clinical samples. $\mathrm{CZ}, \mathrm{KM}, \mathrm{KG}, \mathrm{SZ}, \mathrm{CC}$, $\mathrm{BD}, \mathrm{CP}, \mathrm{WL}$ and $\mathrm{ZY}$ performed the experiments. CZ, RL, LW and KM analyzed the data. CZ, LW and RL drafted the manuscript. All authors read and approved the final manuscript.

\section{Ethics approval and consent to participate}

The present study was approved by the Ethics Committee of the Second Affiliated Hospital of Dalian Medical University (approval no. 2019.087). Written informed consent was obtained from all participants.

\section{Patient consent for publication}

Not applicable.

\section{Competing interests}

The authors declare that they have no competing interests.

\section{References}

1. Gomaa AI, Khan SA, Toledano MB, Waked I and Taylor-Robinson SD: Hepatocellular carcinoma: Epidemiology, risk factors and pathogenesis. World J Gastroenterol 14 4300-4308, 2008.

2.El-Serag HB and Rudolph KL: Hepatocellular carcinoma: Epidemiology and molecular carcinogenesis. Gastroenterology 132: 2557-2576, 2007.

3. Han ZG: Functional genomic studies: Insights into the pathogenesis of liver cancer. Annu Rev Genomics Hum Genet 13: 171-205, 2012

4. Kannan M, Jayamohan S, Moorthy RK, Chabattula SC Ganeshan M and Arockiam AJV: AEG-1/miR-221 axis cooperatively regulates the progression of hepatocellular carcinoma by targeting PTEN/PI3K/AKT signaling pathway. Int J Mol Sci 20: $5526,2019$.

5. Feitelson MA, Arzumanyan A, Kulathinal RJ, Blain SW, Holcombe RF, Mahajna J, Marino M, Martinez-Chantar ML, Nawroth R, Sanchez-Garcia I, et al: Sustained proliferation in cancer: Mechanisms and novel therapeutic targets. Semin Cancer Biol 35 (Suppl): S25-S54, 2015.

6. Christen B, Beck CW, Lombardo A and Slack JM Regeneration-specific expression pattern of three posterior Hox genes. Dev Dyn 226: 349-355, 2003.

7. de Bessa Garcia SA, Araujo M, Pereira T, Mouta J and Freitas R HOX genes function in breast cancer development. Biochim Biophys Acta Rev Cancer 1873: 188358, 2020.

8. Adato O, Orenstein Y, Kopolovic J, Juven-Gershon T and Unger R: Quantitative analysis of differential expression of HOX genes in multiple cancers. Cancers (Basel) 12: 1572, 2020.

9. Anbazhagan R and Raman V: Homeobox genes: Molecular link between congenital anomalies and cancer. Eur J Cancer 33: 635-637, 1997.

10. Argiropoulos B and Humphries RK: Hox genes in hematopoiesis and leukemogenesis. Oncogene 26: 6766-6776, 2007.

11. Li B, Huang Q and Wei GH: The role of HOX transcription factors in cancer predisposition and progression. Cancers (Basel) 11: 528, 2019.

12. Guan Y, He Y, Lv S, Hou X, Li L and Song J: Overexpression of HOXC10 promotes glioblastoma cell progression to a poor prognosis via the PI3K/AKT signalling pathway. J Drug Target 27: 60-66, 2019.

13. Tang XL, Ding BX, Hua Y, Chen H, Wu T, Chen ZQ and Yuan CH: HOXC10 promotes the metastasis of human lung adenocarcinoma and indicates poor survival outcome. Front Physiol 8: 557, 2017.
14. Sadik H, Korangath P, Nguyen NK, Gyorffy B, Kumar R, Hedayati M, Teo WW, Park S, Panday H, Munoz TG, et al: HOXC10 expression supports the development of chemotherapy resistance by fine tuning DNA repair in breast cancer cells. Cancer Res 76: 4443-4456, 2016.

15. Pathiraja TN, Nayak SR, Xi Y, Jiang S, Garee JP, Edwards DP, Lee AV, Chen J, Shea MJ, Santen RJ, et al: Epigenetic reprogramming of HOXC10 in endocrine-resistant breast cancer. Sci Transl Med 6: 229ra41, 2014.

16. Feng X, Li T, Liu Z, Shi Y and Peng Y: HOXC10 up-regulation contributes to human thyroid cancer and indicates poor survival outcome. Mol Biosyst 11: 2946-2954, 2015.

17. Guo C, Hou J, Ao S, Deng X and Lyu G: HOXC10 up-regulation promotes gastric cancer cell proliferation and metastasis through MAPK pathway. Chin J Cancer Res 29: 572-580, 2017.

18. Yao S, He L, Zhang Y, Ye L, Lai Y, Huang L, Wu L, Wu G and Zhu S: HOXC10 promotes gastric cancer cell invasion and migration via regulation of the $N F-\kappa B$ pathway. Biochem Biophys Res Commun 501: 628-635, 2018.

19. Xiong W, Zhou Q, Liu G, Liu XS and Li XY: Homeodomain-containing gene 10 inhibits cell apoptosis and promotes cell invasion and migration in osteosarcoma cell lines. Tumour Biol 39: 1010428317697566, 2017.

20. Livak KJ and Schmittgen TD: Analysis of relative gene expression data using real-time quantitative PCR and the 2(-Delta Delta C(T)) method. Methods 25: 402-408, 2001.

21. Xie F, Li Y, Wang M, Huang C, Tao D, Zheng F, Zhang H, Zeng F, Xiao X and Jiang G: Circular RNA BCRC-3 suppresses bladder cancer proliferation through $\mathrm{miR}-182-5 \mathrm{p} / \mathrm{p} 27$ axis. Mol Cancer 17: 144, 2018.

22. Minagawa M, Ikai I, Matsuyama Y, Yamaoka Y and Makuuchi M: Staging of hepatocellular carcinoma: Assessment of the Japanese TNM and AJCC/UICC TNM systems in a cohort of 13,772 patients in Japan. Ann Surg 245: 909-922, 2007.

23. Wagner EF and Nebreda AR: Signal integration by JNK and p38 MAPK pathways in cancer development. Nat Rev Cancer 9: 537-549, 2009.

24. Hanahan D and Weinberg RA: Hallmarks of cancer: The next generation. Cell 144: 646-674, 2011.

25. Hanahan D and Weinberg RA: The hallmarks of cancer. Cell 100 57-70, 2000.

26. Cillo C, Schiavo G, Cantile M, Bihl MP, Sorrentino P, Carafa V, D' Armiento M, Roncalli M, Sansano S, Vecchione R, et al: The HOX gene network in hepatocellular carcinoma. Int $\mathrm{J}$ Cancer 129: 2577-2587, 2011.

27. Kim J, Piao HL, Kim BJ, Yao F, Han Z, Wang Y, Xiao Z, Siverly AN, Lawhon SE, Ton BN, et al: Long noncoding RNA MALAT1 suppresses breast cancer metastasis. Nat Genet 50: 1705-1715, 2018.

28. Kim J, Yu L, Chen W, Xu Y, Wu M, Todorova D, Tang Q, Feng B Jiang L, He J, et al: Wild-type p53 promotes cancer metabolic switch by inducing PUMA-dependent suppression of oxidative phosphorylation. Cancer Cell 35: 191-203.e8, 2019.

29. Gregory RI and Shiekhattar R: MicroRNA biogenesis and cancer. Cancer Res 65: 3509-3512, 2005.

30. Zhang Y, Tang C, Yu T, Zhang R, Zheng $\mathrm{H}$ and Yan W: MicroRNAs control mRNA fate by compartmentalization based on 3' UTR length in male germ cells. Genome Biol 18: 105, 2017.

31. Lu J, Getz G, Miska EA, Alvarez-Saavedra E, Lamb J, Peck D, Sweet-Cordero A, Ebert BL, Mak RH, Ferrando AA, et al: MicroRNA expression profiles classify human cancers. Nature 435: 834-838, 2005.

32. Peluso I, Yarla NS, Ambra R, Pastore G and Perry G: MAPK signalling pathway in cancers: Olive products as cancer preventive and therapeutic agents. Semin Cancer Biol 56: 185-195, 2019.

33. Ki MR, Lee HR, Goo MJ, Hong IH, Do SH, Jeong DH, Yang HJ, Yuan DW, Park JK and Jeong KS: Differential regulation of ERK1/2 and p38 MAP kinases in VacA-induced apoptosis of gastric epithelial cells. Am J Physiol Gastrointest Liver Physiol 294: G635-G647, 2008.

34. Kim EK and Choi EJ: Compromised MAPK signaling in human diseases: An update. Arch Toxicol 89: 867-882, 2015.

This work is licensed under a Creative Commons Attribution-NonCommercial-NoDerivatives 4.0 International (CC BY-NC-ND 4.0) License. 